\title{
A Continuous Analogue of the Upper Bound Theorem
}

\author{
U. Wagner and E. Welzl \\ Institut für Theoretische Informatik, ETH Zürich, \\ ETH Zentrum, CH-8092 Zürich, Switzerland \\ \{uli,emo\}@inf.ethz.ch
}

\begin{abstract}
For an absolutely continuous probability measure $\mu$ on $\mathbb{R}^{d}$ and a nonnegative integer $k$, let $\tilde{s}_{k}(\mu, \boldsymbol{0})$ denote the probability that the convex hull of $k+d+1$ random points which are i.i.d. according to $\mu$ contains the origin $\mathbf{0}$. For $d$ and $k$ given, we determine a tight upper bound on $\tilde{s}_{k}(\mu, \mathbf{0})$, and we characterize the measures in $\mathbb{R}^{d}$ which attain this bound. As we will see, this result can be considered a continuous analogue of the Upper Bound Theorem for the maximal number of faces of convex polytopes with a given number of vertices. For our proof we introduce so-called $h$-functions, continuous counterparts of $h$-vectors of simplicial convex polytopes.
\end{abstract}

\section{Introduction}

Suppose we choose $n \geq 3$ random points, $P_{1}, \ldots, P_{n}$, i.i.d. according to the uniform distribution in the unit disk centered at the origin. What is the probability that the convex hull of these points contains the origin?

There is a beautiful way of determining this probability due to Wendel [8], see also [1]: First choose $n$ random points $Q_{1}, \ldots, Q_{n}$ in the disk. For each $i$, set $P_{i}$ to $Q_{i}$ or to $-Q_{i}$ with equal probability $1 / 2$. The points $P_{1}, \ldots, P_{n}$ are again i.i.d. random points in the disk. Independently of the choice of the $Q_{i}$ 's, ${ }^{1}$ there are $2 n$ possibilities to choose the signs for the $P_{i}$ 's such that the origin can be separated from these points by a line (every partition of the $Q_{i}$ 's by a line through the origin gives two such possibilities). Therefore, the probability for the convex hull of the $P_{i}$ 's to contain the origin is precisely $1-2 n / 2^{n}$. A second glance at the proof shows that all we used were the facts that the points were chosen from a distribution that is symmetric about the origin and that certain degeneracies occur with probability zero.

\footnotetext{
${ }^{1}$ Here we ignore degenerate configurations which occur with probability zero.
} 
The corresponding probability of $n>d$ random points drawn from such a centrally symmetric distribution guaranteeing almost sure general position on $\mathbb{R}^{d}$ can be shown to be $\mathrm{be}^{2}$

$$
1-\frac{\sum_{i=0}^{d-1}\left(\begin{array}{c}
n-1 \\
i
\end{array}\right)}{2^{n-1}} .
$$

What happens if we choose the points from some other distribution? To this end, let $\mu$ be an absolutely continuous (a.c.) probability measure on $\mathbb{R}^{d}$, that is, one which has a density function with respect to the Lebesgue measure; $\mu$ is called balanced about a point $p$ if every hyperplane through $p$ equipartitions $\mu$ (by absolute continuity, the hyperplane itself has $\mu$-measure zero).

For a nonnegative integer $k$, let $\tilde{s}_{k}(\mu, \mathbf{0})$ be the probability that the convex hull of $k+d+1$ independent and identically $\mu$-distributed random points (i.i.d. $\sim \mu$, for short) contains the origin $\mathbf{0}$. In this paper we show that $\tilde{s}_{n-d-1}(\mu, \mathbf{0})$ is at most the number given in (1). Moreover, this value is attained iff $\mu$ is balanced about the origin. To the best of our knowledge, this result is new. However, as we see it, the main contribution of this paper is the presentation of this result as a continuous analogue of the Upper Bound Theorem, and the introduction of continuous twins of $h$-vectors of convex polytopes, and of $j$-facets of finite point sets. We also discuss continuous versions of the Dehn-Sommerville relations.

For the proof of the bound on $\tilde{s}_{k}(\mu, \mathbf{0})$ we choose a detour by first analyzing the probability that a line intersects the convex hull of $n$ random points (from some probability distribution $\mu$ ). These findings can then be transferred by an appropriate projection to the setting as described above. We begin by preparing the central notions for our presentation.

\subsection{Directed Lines Entering Oriented Simplices}

An oriented simplex $\sigma$ in $\mathbb{R}^{d}$ is a pair $\left(|\sigma|, \mathcal{H}^{+}(\sigma)\right)$, where $|\sigma|$ is an ordinary $(d-1)$ simplex and $\mathcal{H}^{+}(\sigma)$ is one of the components of $\mathbb{R}^{d} \backslash$ aff $(|\sigma|)$ marked as the positive side or open positive halfspace of $\sigma$. Correspondingly, the other component is called the open negative halfspace and denoted by $\mathcal{H}^{-}(\sigma)$. If the positive and the negative halfspaces exchange their roles, we speak of the simplex with reversed orientation, $-\sigma$.

In dimension $d \geq 2$, an orientation of the simplex spanned by affinely independent points $p_{1}, \ldots, p_{d}$ is given in a natural way by the order in which the points come: Defining $\mathcal{H}^{+}\left(p_{1}, \ldots, p_{d}\right)$ to be the set of points $q \in \mathbb{R}^{d}$ with

$$
\operatorname{det}\left[\begin{array}{cccc}
1 & 1 & \cdots & 1 \\
q & p_{1} & \cdots & p_{n}
\end{array}\right]>0,
$$

we obtain the oriented simplex

$$
\left[p_{1}, \ldots, p_{d}\right]:=\left(\operatorname{conv}\left\{p_{1}, \ldots, p_{d}\right\}, \mathcal{H}^{+}\left(p_{1}, \ldots, p_{d}\right)\right)
$$

\footnotetext{
${ }^{2}$ Note that the numerator on the right-hand side is exactly the number of topes (or regions) in an arrangement of $n-1$ affine hyperplanes in dimension $d-1$.
} 
Thus, $\left[p_{1}, \ldots, p_{d}\right]=\left[q_{1}, \ldots, q_{d}\right]$ iff $q_{i}=p_{\pi(i)}, 1 \leq i \leq d$, for an even permutation $\pi$, and $\left[q_{1}, \ldots, q_{d}\right]=-\left[p_{1}, \ldots, p_{d}\right]$ if $\pi$ is odd. In dimension one, however, in addition to a point $p_{1} \in \mathbb{R}$, a sign $\varepsilon \in\{+1,-1\}$ is needed; we then write $\varepsilon\left[p_{1}\right]$ for the oriented simplex $\sigma$ with $|\sigma|=\left\{p_{1}\right\}$ and $\mathcal{H}^{+}(\sigma)=\left\{q \in \mathbb{R} \mid \varepsilon \cdot\left(p_{1}-q\right)>0\right\}$.

Some more terminology: A directed line $\ell$ is said to enter an oriented simplex $\sigma$ if it intersects the relative interior of $|\sigma|$ in a single point and is directed from the positive to the negative side of $\sigma ; \ell$ is said to leave $\sigma$ if it is directed the other way. By contrast, we say that $\ell$ passes into a nonoriented closed subset $\mathcal{C}$ of $\mathbb{R}^{d}$ at $p$ if $p$ is the first point of intersection of $\ell$ with $\mathcal{C}$ (provided such a point exists). Conversely, $\ell$ exits $\mathcal{C}$ at $q$ if $q$ is the last point of intersection. Thus, $\ell$ enters an oriented simplex $\sigma$ if it passes into the closed negative halfspace $\overline{\mathcal{H}^{-}}(\sigma)$ at some point in the relative interior of $\sigma$.

\section{2. $y$-Facets and h-Functions}

We now fix a directed line $\ell$ and an a.c. probability measure $\mu$ in $\mathbb{R}^{d}, d \geq 1$. An oriented simplex $\sigma$ is called a $y$-facet of $\mu$, for a real number $0 \leq y \leq 1$, if $\mu\left(\mathcal{H}^{+}(\sigma)\right)=y$. We will need a vast supply of random points; let $P_{1}, P_{2}, P_{3}, \ldots \in \mathbb{R}^{d}$ be i.i.d. $\sim \mu$. One of the geometrical niceties of absolute continuity is that various special cases are negligible since they only occur with probability zero. For instance, the points are almost surely (a.s.) in general position with respect to $\ell$, and so on. Apart from sporadic reminders, we are pretty much going to ignore such negligible events from now on.

Moreover, allow us to introduce the notion of a $\mu$-random oriented simplex. In dimension $d \geq 2$, this is just the oriented simplex $\sigma=\left[P_{1}, \ldots, P_{d}\right]$; in dimension one, such a random simplex $\sigma=\varepsilon\left[P_{1}\right]$ is obtained by independently choosing $\varepsilon$ as -1 and +1 with equal probability. We also refer to $\sigma$ as the random simplex spanned by $P_{1}, \ldots, P_{d}$. Let then

$$
H(y)=H_{\mu, \ell}(y):=\operatorname{Prob}\left[\ell \text { enters } \sigma \text { and } \mu\left(\mathcal{H}^{+}(\sigma)\right) \leq y\right],
$$

where $\sigma$ is a $\mu$-random oriented simplex. ${ }^{3}$ Obviously, the function $H$ is right continuous and monotone. As a matter of fact, monotonicity suffices to ensure that its derivative, the $h$-function

$$
h(y)=h_{\mu, \ell}(y):=\frac{\mathrm{d} H(y)}{\mathrm{d} y}
$$

is defined almost everywhere (a.e.), that is, everywhere except possibly on some set of Lebesgue measure zero. ${ }^{4}$ There is, however, an issue here that must not be swept under

\footnotetext{
${ }^{3}$ Of course, if we want to measure probabilities, we need to check measurability. First observe that the set $U_{n}^{d}$ consisting of all $n$-tuples of points in general position in $\mathbb{R}^{d}$ is an open subset of $\mathbb{R}^{n \cdot d}$ (small perturbations of the points do not affect general position) and therefore measurable; the same applies to the subset $\left\{\left(p_{i}\right) \in U_{d}^{d} \mid \ell\right.$ enters $\left.\left[p_{1}, \ldots, p_{d}\right]\right\}$ of $U_{d}^{d}$. Moreover, by absolute continuity of $\mu$,

$$
\left(p_{1}, \ldots, p_{d}\right) \mapsto \mu\left(\mathcal{H}^{+}\left(p_{1}, \ldots, p_{d}\right)\right)
$$

is a continuous, hence measurable, function on $U_{d}^{d}$. Therefore, $\{\ell$ enters $\sigma\}$ is an event, i.e., a measurable subset of the underlying probability space, and $\mu\left(\mathcal{H}^{+}(\sigma)\right)$ is a random variable.

${ }^{4}$ That monotone functions are a.e. differentiable is a special case of Lebesgue's Differentiation Theorem (see [3]).
} 
the carpet. Namely, from monotonicity and differentiability a.e. alone we cannot infer that $H(y)=\int_{0}^{y} h(x) \mathrm{d} x .^{5}$ In fact, for a function $F:[a, b] \rightarrow \mathbb{R}$ on a compact interval, the following are equivalent (see Section 3.5 of [3]):

1. There is some Lebesgue integrable function $f$ on $[a, b]$ such that $F(x)-F(a)=$ $\int_{a}^{x} f(x) \mathrm{d} x$ for all $x \in[a, b]$.

2. $F$ is absolutely continuous ${ }^{6}$ in the sense that for all $\varepsilon>0$, there exists some $\delta>0$ such that for any finite set of disjoint open intervals $\left(a_{1}, b_{1}\right) \cdots\left(a_{k}, b_{k}\right) \subseteq[a, b]$,

$$
\sum_{j=1}^{k}\left(b_{j}-a_{j}\right)<\delta \Rightarrow \sum_{j=1}^{k}\left|F\left(b_{j}\right)-F\left(a_{j}\right)\right|<\varepsilon .
$$

If these conditions are satisfied, $F$ is differentiable a.e. on $[a, b]$ and $(\mathrm{d} / \mathrm{d} x) F(x)=f(x)$ for a.e. $a \leq x \leq b$. We will see in Section 3 that $H$ is even continuously differentiable, which will resolve our worries. However, for the time being, we must not assume this.

In Section 4 we briefly indicate how $h$-functions correspond to the $h$-vectors of convex polytopes. First and foremost, however, we prove analogues of some properties of $h$ vectors, notably

$$
h(y)=h(1-y),
$$

in analogy to the Dehn-Sommerville relations, and

$$
h(y) \leq \frac{d}{2} \min \left\{y^{d-1},(1-y)^{d-1}\right\},
$$

in analogy to the Upper Bound Theorem. Note that once we have established absolute continuity of $H$, this yields an upper bound of $1 / 2^{d}$ on $H(1)=\int_{0}^{1} h(y) \mathrm{d} y$, the probability that a random oriented simplex is entered by $\ell$. It follows that an undirected line intersects the convex hull of $d$ random (according to $\mu$ ) points with probability at most $1 / 2^{d-1}$. By an appropriate lifting from $\mathbb{R}^{d}$ to $\mathbb{R}^{d+1}$, we may conclude $\tilde{s}_{1}(\mu, \mathbf{0}) \leq 1 / 2^{d}$ for an absolutely continuous probability distribution $\mu$ in $\mathbb{R}^{d}, d \geq 1$. We will see in Section 2 how the moments of the distribution defined by $H$ yield the bounds (1) for $\tilde{s}_{k}(\mu, \mathbf{0}), k \geq 0$.

\section{Moments and the Dehn-Sommerville Equation}

As a warm-up, we consider the one-dimensional case. Suppose first that $\ell$ is directed from the right to the left. Then a random simplex $\varepsilon[P]$ is entered by $\ell$ if and only if $\varepsilon=-1$. Therefore ${ }^{7} H(y)=\frac{1}{2} \operatorname{Prob}[\mu((P, \infty)) \leq y]=y / 2$, by monotonicity of $H$ and continuity of $p \mapsto \mu((p, \infty))$. By parity of reasoning for the case where $\ell$ is directed from left to right, we conclude:

\footnotetext{
5 The Cantor function, for instance, furnishes an example of a monotone and even continuous function which maps $[0,1]$ onto itself while its derivative vanishes a.e. (see [3]).

${ }^{6}$ The resemblance of terminology is no coincidence, absolute continuity of functions and of measures are closely related (see [3]).

${ }^{7}$ Observe that $(P, \infty)$ will denote an interval.
} 
Observation 2.1. In dimension one,

$$
H(y)=\frac{y}{2} \quad \text { and } \quad h(y)=\frac{1}{2} .
$$

Trivial as these observations are, they provide a nice base case for inductive reasoning, which we employ repeatedly in the following.

\subsection{Moments}

Apart from the fact that $H(1)<1, H$ behaves just like a probability distribution function on the unit interval: it is nonnegative, monotonically increasing, and right continuous. ${ }^{8}$ As in the case of probability distributions, we define, for integer $k \geq 0$, the $k$ th moment of $H$ to be the Lebesgue-Stieltjes integral with respect to $H$ of $y^{k}$,

$$
M_{k}(H):=\int_{0}^{1} y^{k} \mathrm{~d} H(y) .
$$

What we would like to write instead is $\int_{0}^{1} y^{k} h(y) \mathrm{d} y$, and that is what you may wish to think of when reading (6); however, it would be dishonest to use the more familiar notation at this point, for it is only correct provided that $H$ is a.c. Yet in order to prove the latter, the apparatus of abstract integration is needed if we do not want to beg the question.

More generally, it makes sense to speak of moments for a wider class of functions which contains $H$. More precisely, we call a function $F:[a, b] \rightarrow \mathbb{R}$ nice if it can be written as a difference $F=F_{1}-F_{2}$ of right continuous, bounded monotone functions. The point of considering moments is that a nice function $F$ on a compact interval has moments of all orders and that, moreover, these uniquely determine $F$ :

Lemma 2.2. If $F, G:[a, b] \rightarrow \mathbb{R}$ are nice functions such that $M_{k}(F)=M_{k}(G)$ for all $k \geq 0$, then $F=G$.

(The case of probability distribution functions, i.e., of monotone functions for which the 0th moments equal 1, is proved in Section 2.3.e of [2]. The general case follows by linearity. ${ }^{9}$ )

Thus, while it may at first seem difficult to pin down any interesting properties of $H$ or $h$, we can try to get a grip on their moments. Of course, $M_{k}(H)=1 / 2(k+1)$ in

\footnotetext{
${ }^{8}$ In fact, $H$ is the distribution function of a finite measure $\eta$ on $[0,1]$ which is given by $\eta([0, y])=H(y)$; we could make $\eta$ into a probability by normalizing by the constant factor $1 / H(1)$, that is, by considering conditional probabilities in the definition of $H$.

${ }^{9}$ That is, by the fact that for all $\left.c_{1}, c_{2} \in \mathbb{R}, M_{k}\left(c_{1} F_{1}+c_{2} F_{2}\right)=c_{1} M_{k}\left(F_{1}\right)+c_{2} M_{k}\left(F_{2}\right)\right)$ : Namely, if $F=F_{1}-F_{2}$ and $G=G_{1}-G_{2}$, then $M_{k}\left(F_{1}\right)-M_{k}\left(F_{2}\right)=M_{k}(F)=M_{k}(G)=M_{k}\left(G_{1}\right)-M_{k}\left(G_{2}\right)$. Consider $c:=M_{0}\left(F_{1}+G_{2}\right)=M_{0}\left(F_{2}+G_{1}\right)$. If $c=0$, then, by monotonicity, $F_{1}+G_{2}$ and $F_{2}+G_{1}$ are constant on $[a, b]$, say $F_{1}+G_{2}=r$ and $F_{2}+G_{1}=s$. Then $r(b-a)=M_{1}\left(F_{1}+G_{2}\right)=M_{1}\left(F_{2}+G_{1}\right)=s(b-a)$, hence $r=s$, hence $F=G$. If, on the other hand, $c \neq 0$, then $M_{k}(\bar{F})=M_{k}(\bar{G})$ for all $k$, where $\bar{F}=\left(F_{1}+G_{2}\right) / c$ and $\bar{G}=\left(F_{2}+G_{1}\right) / c$. However, these are probability distribution functions, hence $\bar{F}=\bar{G}$, hence $F=G$.
} 
dimension one. Hence, assume $d \geq 2$ until further notice. Intuitively speaking, if $\sigma=$ $\left[P_{1}, \ldots, P_{d}\right]$ is a $y$-facet, then by independence $y^{k}=\operatorname{Prob}\left[P_{d+1}, \ldots, P_{d+k} \in \mathcal{H}^{+}(\sigma)\right]$, and by averaging over all $y$, we arrive at

\section{Observation 2.3.}

$$
M_{k}(H)=\operatorname{Prob}\left[\ell \text { enters } \sigma \text { and } P_{d+1}, \ldots, P_{d+k} \in \mathcal{H}^{+}(\sigma)\right] \text {. }
$$

Proof. For $d \geq 2$, the transformation theorem for image measures yields

$$
\begin{aligned}
\int_{0}^{1} y^{k} \mathrm{~d} H(y)= & \int_{\left\{\left(p_{i}\right)_{i=1}^{d} \mid \ell \text { enters }\left[p_{1}, \ldots, p_{d}\right]\right\}} \cdots \int_{\substack{\left\{\left(p_{i}\right)_{i=1}^{d+k} \mid \ell \operatorname{lenters}\left[p_{1}, \ldots, p_{d}\right] \text { and } \\
p_{d+1}, \ldots, p_{d+k} \in \mathcal{H}^{+}\left(p_{1}, \ldots, p_{d}\right)\right\}}} \mu\left(\mathcal{H}^{+}\left(p_{1}, \ldots, p_{d}\right)\right)^{k} \mathrm{~d} \mu\left(p_{d}\right) \cdots \mathrm{d} \mu\left(p_{1}\right) \\
= & \operatorname{Prob}\left[\ell \text { enters } \sigma=\left[P_{1}, \ldots, P_{d}\right] \text { and } P_{d+1} \ldots P_{d+k} \in \mathcal{H}^{+}(\sigma)\right],
\end{aligned}
$$

by independence of the $P_{i}$ 's.

It is convenient to modify the geometric interpretation (7) further so as to make it more "symmetric." The following lemma is a useful tool:

Lemma 2.4 (Counting Permutations). Suppose $\boldsymbol{X}=\left(X_{1}, \ldots, X_{n}\right)$ is a vector of i.i.d. random variables which take values in some set $N$, and let $A$ and $B$ be measurable sets of $n$-tuples $\left(x_{i}\right)_{i=1}^{n}$ of elements in $N$. Assume furthermore that $l, m$ are constants such that for every $\boldsymbol{x}=\left(x_{i}\right) \in A$ and every $\boldsymbol{x}^{\prime}=\left(x_{i}^{\prime}\right) \in B$, there are exactly l permutations $\pi$ such that $\boldsymbol{x}_{\pi}:=\left(x_{\pi(i)}\right) \in B$, and exactly m permutations $\pi^{\prime}$ for which $\boldsymbol{x}_{\pi^{\prime}}^{\prime} \in A$. Then

$$
l \cdot \operatorname{Prob}[\boldsymbol{X} \in A]=m \cdot \operatorname{Prob}[\boldsymbol{X} \in B] .
$$

Proof. Let $\Pi$ be a random permutation on $n$ letters, chosen independently of the $X_{i}$ 's, every permutation being equally probable. By assumption, if $x \in A$, then $\operatorname{Prob}\left[x_{\Pi} \in B\right]$ $=l / n$ !. Since this holds for all $\boldsymbol{x} \in A$ and since $\boldsymbol{X}$ and $\Pi$ are independent, we get Prob $\left[\boldsymbol{X}_{\Pi} \in B \mid \boldsymbol{X} \in A\right]=l / n$ !, i.e.,

$$
\frac{l}{n !} \operatorname{Prob}[\boldsymbol{X} \in A]=\operatorname{Prob}\left[\boldsymbol{X}_{\Pi} \in B \text { and } \boldsymbol{X} \in A\right] .
$$

However, by independence, the random vector $\boldsymbol{X}_{\Pi}$ has the same distribution as $\boldsymbol{X}$; therefore, the right-hand side of (8) equals

$$
\operatorname{Prob}\left[\boldsymbol{X} \in B \text { and } \boldsymbol{X}_{\Pi^{-1}} \in A\right]=\frac{m}{n !} \operatorname{Prob}[\boldsymbol{X} \in B],
$$

as above, since $\Pi^{-1}$ is again a random permutation. 
Lemma 2.5. Let $s_{k}=s_{k}(\mu, \ell)$ stand for the probability that $\ell$ intersects the convex hull of $P_{1}, \ldots, P_{d+k}$. Then

$$
M_{k}(H)=\frac{1}{2\left(\begin{array}{c}
d+k \\
d
\end{array}\right)} s_{k} .
$$

Proof. Let $B$ denote the set of all $(d+k)$-tuples $\left(p_{i}\right)_{i=1}^{d+k}$ of points in general position in $\mathbb{R}^{d}$ such that $\ell$ intersects $\operatorname{conv}\left\{p_{1}, \ldots, p_{d+k}\right\}$, and set $A:=\left\{\left(p_{i}\right) \in B \mid \ell\right.$ enters $\left[p_{1}, \ldots, p_{d+k}\right]$ and $\left.p_{d+1}, \ldots, p_{d+k} \in \mathcal{H}^{+}\left(p_{1}, \ldots, p_{d}\right)\right\}$. Then, for $P_{1}, \ldots, P_{d+k}$ i.i.d. $\sim \mu$ as above, $M_{k}=\operatorname{Prob}\left[\left(P_{i}\right) \in A\right]$ (recall that the general position is for free, by absolute continuity of $\mu$, and, moreover, $s_{k}=\operatorname{Prob}\left[\left(P_{i}\right) \in B\right]$. In the spirit of Lemma 2.4, we count permutations. Obviously, if $\left(p_{i}\right) \in A$, then $\left(p_{\pi(i)}\right) \in B$ for every permutation $\pi$ on $d+k$ letters. Conversely, assume $\left(p_{i}\right) \in B$. By general position, the convex hull of the $p_{i}$ 's is a simplicial polytope $\mathcal{P}$; in particular, the facet of $\mathcal{P}$ through which $\ell$ exits $\mathcal{P}$ is a simplex $|\sigma|$. There are exactly $d$ ! $k$ ! permutations $\pi$ of the $p_{i}$ 's such that $|\sigma|=\operatorname{conv}\left\{p_{\pi(1)}, \ldots, p_{\pi(d)}\right\}$, and one-half of these give that simplex the right orientation, such that $\ell$ is oriented from the positive to the negative side of the resulting oriented simplex $\sigma=\left[p_{\pi(1)}, \ldots, p_{\pi(d)}\right]$. However, since $\ell$ exits $\mathcal{P}$ through $|\sigma|$, this is equivalent to saying that the remaining points $p_{\pi(d+1)}, \ldots, p_{\pi(d+k)}$ lie on the positive side of $\sigma$. It follows by Lemma 2.4 that

$$
(d+k) ! \operatorname{Prob}\left[\left(P_{i}\right) \in A\right]=\frac{d ! k !}{2} \operatorname{Prob}\left[\left(P_{i}\right) \in B\right],
$$

which is what we wished to show.

Evidently, the probability that $\ell$ intersects $\operatorname{conv}\left\{P_{1}, \ldots, P_{d+k}\right\}$ does not depend on the orientation of $\ell$; thus, $s_{k}(\mu, \ell)=s_{k}(\mu,-\ell)$ for all $k \in \mathbb{N}_{0}$. However, (9) tells us that $H$ is uniquely determined by the $s_{k}$ 's, hence $H_{\mu, \ell}=H_{\mu,-\ell}$, and therefore

Theorem 2.6 (Dehn-Sommerville Equation). $\quad H(y)=H(1)-H(1-y)$ for $0 \leq$ $y \leq 1$. In terms of $h$, this reads

$$
h(y)=h(1-y)
$$

(Observe that until continuity of $h$ is established, we only know that (10) holds a.e.)

Proof. For $d=1$, this holds by (5); and for $d \geq 2$, the above considerations show that

$$
\begin{aligned}
H_{\mu, \ell}(y) & =H_{\mu,-\ell}(y) \\
& =\operatorname{Prob}\left[\ell \text { leaves }\left[P_{1}, P_{2}, \ldots, P_{d}\right] \text { and } \mu\left(\mathcal{H}^{+}\left(P_{1}, P_{2}, \ldots, P_{d}\right)\right) \leq y\right] \\
& =\operatorname{Prob}\left[\ell \text { enters }\left[P_{2}, P_{1}, \ldots, P_{d}\right] \text { and } \mu\left(\mathcal{H}^{+}\left(P_{2}, P_{1}, \ldots, P_{d}\right)\right)>1-y\right] \\
& =H_{\mu, \ell}(1)-H_{\mu, \ell}(y) .
\end{aligned}
$$




\subsection{Liftings and Projections}

We return to the probabilities $\tilde{s}_{k}(\mu, \mathbf{0})=\operatorname{Prob}\left[\mathbf{0} \in \operatorname{conv}\left\{P_{1}, \ldots, P_{d+1+k}\right\}\right]$ mentioned in the Introduction and see how they relate to $h$-functions. In order to do this, we drop the assumption $d \geq 2$ and lift the set-up to dimension $d+1$ as follows:

Take your favorite a.c. probability distribution on the real line, for instance, the uniform distribution on some bounded interval, and pick, for each $i \in \mathbb{N}$, a $(d+1)$ st coordinate $X_{i} \in \mathbb{R}$ according to that distribution. ${ }^{10}$ Then the lifted points $\tilde{P}_{i}:=\left(P_{i}, X_{i}\right)$ are i.i.d. according to some a.c. probability measure $\tilde{\mu}$ on $\mathbb{R}^{d+1}$. If we take $\tilde{\ell}$ to be the $x_{d+1}$-axis, then

$$
\tilde{s}_{k}(\mu, \mathbf{0})=s_{k}(\tilde{\mu}, \tilde{\ell})
$$

(observe that this does not depend on the distribution of the $(d+1)$ st coordinate), and we just write $\tilde{s}_{k}$ from now on. On the one hand, this implies that any bound for $h_{\tilde{\mu}, \tilde{\ell}}$ immediately gives a bound for $\tilde{s}_{k}=2\left(\begin{array}{c}d+k+1 \\ k\end{array}\right) \int_{0}^{1} y^{k} h_{\tilde{\mu}, \tilde{\ell}}(y) \mathrm{d} y$ (assuming absolute continuity of $H_{\tilde{\mu}, \tilde{\ell}}$.

On the other hand, it opens up a door for induction. Namely, suppose that conversely, we are given an a.c. probability measure $\tilde{\mu}$, a directed line $\tilde{\ell}$, and random points $\tilde{P}_{1}, \tilde{P}_{2}, \tilde{P}_{3}, \ldots$ i.i.d. $\sim \tilde{\mu}$ in $\mathbb{R}^{d+1}$. Up to an affine coordinate transformation, which preserves absolute continuity and does not affect $H_{\tilde{\mu}, \tilde{\ell}}$, we may assume that $\tilde{\ell}$ is the $x_{d+1}$-axis. We identify $\mathbb{R}^{d}$ with $\mathbb{R}^{d} \times\{0\} \subset \mathbb{R}^{d+1}$ and define $P_{i}$ to be the orthogonal projection onto $\mathbb{R}^{d}$ of $\tilde{P}_{i}$. Hence $P_{1}, P_{2}, P_{3}, \ldots \in \mathbb{R}^{d}$ are i.i.d. according to the image $\mu$ of $\tilde{\mu}$ under that projection, and $\mu$ is a.c. again. The above considerations show that the "moments" $\tilde{s}_{k}$, and thereby $H_{\tilde{\mu}, \tilde{\ell}}$, are uniquely determined by $\mu$, which lives in one dimension less. The question remains: Where do we get a directed line $\ell \subseteq \mathbb{R}^{d}$ for the induction step? However, that will be answered in the next section.

We conclude this section by generalizing the definition of "balanced" given in the introduction: $\mu$ is balanced about an affine flat $\mathcal{A}$ if every hyperplane containing $\mathcal{A}$ equipartitions $\mu$.

Observation 2.7. The measure $\mu$ is balanced about $\mathbf{0}$ if and only if $\tilde{\mu}$ is balanced about $\tilde{\ell}$.

\section{A Continuous Upper Bound Theorem}

In this section we inductively establish absolute continuity of $H$, i.e., $H(y)=\int_{0}^{y} h(y) \mathrm{d} y$, by expressing the moments of $H$ in terms of quantities which stem from one dimension less. This will pave the way to the continuous version of the Upper Bound Theorem (UBT) and to the promised bounds for $\tilde{s}_{k}(\mu, \mathbf{0})$.

As indicated above, assume for the induction step that we start out with $\tilde{\mu}, \tilde{\ell}$, and $\tilde{P}_{1}, \tilde{P}_{2}, \tilde{P}_{3}, \ldots$ i.i.d. $\sim \tilde{\mu}$ in $\mathbb{R}^{d+1}$, and that $\mu$ and $P_{1}, P_{2}, P_{3}, \ldots$ are obtained by orthogonal projection onto $\mathbb{R}^{d}$ (generally, entities marked with “ " will live in $\mathbb{R}^{d+1}$, those

${ }^{10}$ In such a way that the $X_{j}$ 's and $P_{i}$ 's are all independent. 
without in $\mathbb{R}^{d}$ ). Recall that, without loss of generality, $\tilde{\ell}$ is the $x_{d+1}$-axis, and that in the case $d=1$, there is an additional random sign $\varepsilon \in\{+1,-1\}$ involved in $\mu$-random oriented simplices.

Next, we choose and fix a (completely arbitrary) directed line $\ell \subseteq \mathbb{R}^{d}$ through the origin 0 (recall that $\tilde{\ell}$ projects onto 0). We abbreviate $\tilde{H}:=H_{\tilde{\mu}, \tilde{\ell}}$ and $H:=H_{\mu, \ell}$, and their derivatives as $\tilde{h}$ and $h$, respectively.

\subsection{The $h^{*}$ - and ${ }^{*} h$-Functions}

For $0 \leq y \leq 1$, set

$$
H^{*}(y)=H_{\mu, \ell}^{*}(y):=\operatorname{Prob}\left[\ell \text { enters } \sigma \text { before } \mathbf{0} \text { and } \mu\left(\mathcal{H}^{+}(\sigma)\right) \leq y\right],
$$

where " $\ell$ enters $\sigma$ before $\mathbf{0}$ " means that $\ell$ enters $\sigma$ and $\mathbf{0} \in \mathcal{H}^{-}(\sigma)$; accordingly, we say that " $\ell$ leaves $\sigma$ before $\mathbf{0}$ " if $\ell$ leaves $\sigma$ and $\mathbf{0} \in \mathcal{H}^{+}(\sigma)$, and so on.

Again, the derivative $h^{*}=h_{\mu, \ell}^{*}:=\mathrm{d} H^{*}(y) / \mathrm{d} y$ exists a.e.; but more can be said than this. Inductively, we may assume that $H$ is a.c., i.e., satisfies condition (4). Moreover, $0 \leq H^{*}(y)-H^{*}(x) \leq H(y)-H(x)$ for $x \leq y$; it follows that $H^{*}$ is a.c., too. Hence, $H^{*}$ is the integral of its derivative, which will be called the $h^{*}$-function of $\mu$ and $\ell$. Analogously, ${ }^{*} H$ is defined by replacing "before" by "after" in (12), and thus the * $h$-function is obtained. Note that since almost no $\mu$-random simplex $\sigma$ contains the origin, we have $H(y)=H^{*}(y)+{ }^{*} H(y)$, and therefore

$$
h(y)=h^{*}(y)+{ }^{*} h(y) \text { a.e. }
$$

Observation 3.1. In dimension $d=1$, let $a:=\mu((-\infty, 0))$. If $\ell$ is directed from left to right, then $H^{*}(y)=\frac{1}{2} \min \{y, a\}$, whence

$$
h^{*}(y)= \begin{cases}\frac{1}{2} & \text { fora.e. } \quad 0 \leq y \leq a, \\ 0 & \text { otherwise }\end{cases}
$$

Proof. Recall from Observation 2.1 that in this set-up

$$
H(y)=\frac{1}{2} \operatorname{Prob}[\mu((-\infty, P)) \leq y]=y,
$$

where $P \in \mathbb{R}^{1}$ is a $\mu$-random point (the $\frac{1}{2}$ stems from the fact that the random sign $\varepsilon$ has to be 1). Now, if we impose the additional condition that the simplex $[P]$ be entered before 0 , then we arrive at $H^{*}(y)=\frac{1}{2} \operatorname{Prob}[\mu((-\infty, P)) \leq y$ and $P<0]=\frac{1}{2} \min \{y, a\}$.

We would like to express the $\tilde{s}_{k}$ 's in terms of $h^{*}$. To this end, let $C_{k}$ denote the event that $\ell$ intersects the convex hull $\mathcal{P}_{k}$ of $P_{1}, \ldots, P_{d+k+1}$ and passes into it before $\mathbf{0}$. In this event, there are two cases: either $\ell$ exits $\mathcal{P}_{k}$ after the origin, i.e., $\mathbf{0} \in \mathcal{P}_{k}$, or $\ell$ also exits $\mathcal{P}_{k}$ before the origin. The probability of the first case is just $\tilde{s}_{k}$. Thus, if we write $D_{k}$ for the second case, we get

$$
\tilde{s}_{k}=\operatorname{Prob}\left(C_{k}\right)-\operatorname{Prob}\left(D_{k}\right) .
$$




\section{Lemma 3.2.}

$$
\begin{aligned}
\frac{1}{2\left(\begin{array}{c}
d+1+k \\
d
\end{array}\right)} \operatorname{Prob}\left(C_{k}\right) & =\operatorname{Prob}\left[\ell \text { enters } \sigma \text { before } \mathbf{0} \text { and } P_{d+1}, \ldots, P_{d+k} \in \mathcal{H}^{-}(\sigma)\right] \\
& =\int_{0}^{1}(1-y)^{k+1} h^{*}(y) \mathrm{d} y
\end{aligned}
$$

Proof. We count permutations: Let $B$ be the set of all $(d+k+1)$-tuples $\left(p_{i}\right)$ of points in general position in $\mathbb{R}^{d}$ such that $\ell$ intersects $\operatorname{conv}\left\{p_{1}, \ldots, p_{d+k+1}\right\}$ and passes into it before $\mathbf{0}$, and define

$$
\begin{aligned}
A=\{ & \left(p_{i}\right) \in B \mid \ell \text { enters }\left[p_{1}, \ldots, p_{d}\right] \text { before } \mathbf{0} \\
& \text { and } \left.p_{d+1}, \ldots, p_{d+k+1} \in \mathcal{H}^{-}\left(p_{1}, \ldots, p_{d}\right)\right\} .
\end{aligned}
$$

If $\left(p_{i}\right) \in A$, then any permutation of the $p_{i}$ 's lies in $B$; on the other hand, exactly $\frac{1}{2} d !(k+1)$ ! permutations take an element $\left(p_{i}\right) \in B$ to an element of $A$, since we have to assign the indices $1, \ldots, d$ and the right orientation to the simplicial facet where $\ell$ exits the convex hull of the points, and the remaining indices to the other points. Applying Lemma 2.4, we obtain the first equality, ${ }^{11}$ while the second is proved just like (7).

Similarly, one shows

\section{Lemma 3.3.}

$$
\begin{aligned}
\frac{1}{2\left(\begin{array}{c}
d+1+k \\
d
\end{array}\right)} \operatorname{Prob}\left(D_{k}\right) & =\operatorname{Prob}\left[\ell \text { enters } \sigma \text { before } \mathbf{0} \text { and } P_{d+1}, \ldots, P_{d+1+k} \in \mathcal{H}^{+}(\sigma)\right] \\
& =\int_{0}^{1} y^{k+1} h^{*}(y) \mathrm{d} y
\end{aligned}
$$

\subsection{Assembling the Parts}

The preceding lemmas together with (14) yield

$$
\frac{1}{2\left(\begin{array}{c}
d+1+k \\
d
\end{array}\right)} \tilde{s}_{k}=\int_{0}^{1} y^{k+1}\left(h^{*}(1-y)-h^{*}(y)\right) \mathrm{d} y
$$

and integration by parts $^{12}$ gives

$$
\frac{1}{2\left(\begin{array}{c}
d+1+k \\
d+1
\end{array}\right)} \tilde{s}_{k}=\int_{0}^{1} y^{k}\left((d+1) \int_{0}^{y} h^{*}(x)-h^{*}(1-x) \mathrm{d} x\right) \mathrm{d} y
$$

\footnotetext{
${ }^{11}$ Strictly speaking, the argument as stated is only valid for $d \geq 2$, but the random sign $\varepsilon$ makes the statement come out true in dimension one as well.

12 This is justified since $y \mapsto \int_{0}^{y} h^{*}(1-x)-h^{*}(x) \mathrm{d} x$ is a.c.; see Section 3.5 of [3].
} 
for all $k \in \mathbb{N}_{0}$. However, the expression on the left is exactly the $k$ th moment of $\tilde{H}$, and the integral on the right is that of the nice function given by

$$
y \mapsto \int_{0}^{y}(d+1)\left(H^{*}(x)-H^{*}(1)+H^{*}(1-x)\right) \mathrm{d} x
$$

Since nice functions are completely determined by their moments and since the above integrand is continuous, we conclude

Theorem 3.4. $\tilde{H}$ is continuously differentiable, and

$$
\tilde{h}(y)=(d+1) \int_{0}^{y}\left(h^{*}(x)-h^{*}(1-x)\right) \mathrm{d} x
$$

for $0 \leq y \leq 1$. It follows that $\tilde{h}$ itself is absolutely continuous.

For the tightness part of the upper bound on $h$, we need the following:

Lemma 3.5. Given $\tilde{\mu}$ and $\tilde{\ell}$ in dimension $d+1=2$, let $\tilde{\mathcal{H}}_{\mathrm{L}}$ and $\tilde{\mathcal{H}}_{\mathrm{R}}$ denote the open halfspaces left and right of $\tilde{\ell}$, respectively. If we set $a:=\min \left\{\tilde{\mu}\left(\tilde{\mathcal{H}}_{\mathrm{L}}\right), \tilde{\mu}\left(\tilde{\mathcal{H}}_{\mathrm{R}}\right)\right\}$, then

$$
\tilde{h}(y)=\min \{y, 1-y, a\} .
$$

Proof. As before, we identify $\tilde{\ell}^{\perp}$ with $\mathbb{R}^{1}$ in such a way that $\tilde{\ell}$ orthogonally projects onto 0 , and that $\tilde{\mathcal{H}}_{\mathrm{L}}$ and $\tilde{\mathcal{H}}_{\mathrm{R}}$ project onto the intervals $(-\infty, 0)$ and $(0, \infty)$, respectively. Thus, $a=\min \{\mu((-\infty, 0)), \mu((0, \infty))\}$. Take $\ell=\mathbb{R}^{1}$ to be directed from left to right, say. Then, by (18) and Observation 3.1,

$$
\begin{aligned}
\tilde{h}(y) & =2 \int_{0}^{y} h^{*}(x) \mathrm{d} x-2 \int_{1-y}^{1} h^{*}(x) \mathrm{d} x \\
& =2 \int_{0}^{\min \{y, a\}} \frac{1}{2} \mathrm{~d} x-\int_{\min \{a, 1-y\}}^{a} \frac{1}{2} \mathrm{~d} x \\
& =\min \{y, 1-y, a\},
\end{aligned}
$$

as desired.

Theorem 3.6 (Continuous Upper Bound Theorem (CUBT)). The h-function of $\mu$ and $\ell$ satisfies

$$
h(y) \leq \frac{d}{2} \min \left\{y^{d-1},(1-y)^{d-1}\right\} .
$$

It follows that $H(y) \leq \frac{1}{2} \min \left\{y^{d}, 2^{1-d}-(1-y)^{d}\right\}$. These upper bounds are tight, and equality a.e. is attained if and only if $\mu$ is balanced about the line $\ell$. More precisely, 
if there is a hyperplane $\mathcal{H} \supseteq \ell$ with $\mu\left(\mathcal{H}^{+}\right)=: a<\frac{1}{2}<1-a=\mu\left(\mathcal{H}^{-}\right)$then, for $0 \leq y \leq \frac{1}{2}$,

$$
h(y) \leq \begin{cases}\frac{d}{2} y^{d-1} & \text { if } \quad 0 \leq y \leq a, \text { and } \\ \frac{d}{2}\left(y^{d-1}-(y-a)^{d-1}\right) & \text { if } \quad a \leq y \leq \frac{1}{2} .\end{cases}
$$

That is, $h(y)$ falls strictly short of the upper bound (19) on the interval $(a, 1-a)$.

Proof. Observation 2.1 takes care of the assertions in dimension $d=1$ (note that any measure on $\mathbb{R}^{1}$ is vacuously balanced about the real line itself). Assume then that (19) holds in dimension $d$. To establish it in dimension $d+1$, it suffices to consider $0 \leq y \leq \frac{1}{2}$, by the Dehn-Sommerville equation. We have

$$
h^{*}(x)-h^{*}(1-x) \stackrel{(\mathrm{i})}{\leq} h^{*}(x) \stackrel{\text { (ii) }}{\leq} h^{*}(x)+{ }^{*} h(x)=h(x),
$$

and therefore, by induction (iii),

$$
\tilde{h}(y) \stackrel{(\mathrm{i})+(\text { ii) }}{\leq}(d+1) \int_{0}^{y} h(x) \mathrm{d} x \stackrel{\text { (iii) }}{\leq} \frac{d+1}{2} \int_{0}^{y} \mathrm{~d} x^{d-1} \mathrm{~d} x=\frac{d+1}{2} y^{d} .
$$

Now we turn to the last claim. On the one hand, if $\tilde{\mu}$ is balanced about $\tilde{\ell}$ in $\mathbb{R}^{d+1}$, then the projected measure $\mu$ is balanced about the origin (Observation 2.7). Therefore, by induction, $h(y)=(d / 2) y^{d-1}$. This tells us that the second inequality (iii) in (22) is, in fact, an equality. It remains to prove that (i) and (ii) in (21) are also equalities. However, since $\mu$ is balanced about $\mathbf{0}$, any halfspace $\mathcal{H}$ containing $\mathbf{0}$ has measure $\mu(\mathcal{H}) \geq \frac{1}{2}$. It follows that for $x<\frac{1}{2}$ and $\delta>0$ sufficiently small, no matter which directed line $\ell \subseteq \mathbb{R}^{d}$ through $\mathbf{0}$ we have chosen, there are no $(x \pm \delta)$-facets of $\mu$ which $\ell$ leaves after $\mathbf{0}$, and no $(1-x \pm \delta)$-facets which it enters before $\mathbf{0}$. Letting $\delta \rightarrow 0$, we derive $* h(x)=0=h^{*}(1-x)$ for a.e. $x \leq \frac{1}{2}$. This shows the "if" part of the asserted equivalence.

On the other hand, assume that $\tilde{\mu}$ is not balanced about $\tilde{\ell}$, i.e., that there is some oriented hyperplane $\tilde{\mathcal{H}} \supset \tilde{\ell}$ such that $\tilde{\mu}\left(\tilde{\mathcal{H}}^{+}\right)=: a<\frac{1}{2}<b:=\tilde{\mu}\left(\tilde{\mathcal{H}}^{-}\right)$. In dimension 2 , bound (20) is just Lemma 3.5. If $d+1 \geq 3$, we set $\mathcal{H}:=\tilde{\mathcal{H}} \cap \mathbb{R}^{d}$ and choose the line $\ell$ to be contained in $\mathcal{H}$. Then $\mu$ is unbalanced about $\ell$ since $\mu\left(\mathcal{H}^{+}\right)=a$ and $\mu\left(\mathcal{H}^{-}\right)=b$. Inductively, $h$ satisfies the estimate (20), and (22) guarantees that, mutatis mutandis, so does $\tilde{h}$.

Plugging into $\tilde{s}_{k}=2\left(\begin{array}{c}d+k+1 \\ d+1\end{array}\right) \int_{0}^{1} y^{k} \tilde{h}(y) \mathrm{d} y$ the upper bound which the CUBT provides for the integrand on the right, we get the following:

\section{Corollary 3.7.}

$$
\tilde{s}_{k}(\mu, \mathbf{0})=\operatorname{Prob}\left[\mathbf{0} \in \operatorname{conv}\left\{P_{1}, \ldots, P_{d+k+1}\right\}\right] \leq \frac{\sum_{i=0}^{k}\left(\begin{array}{c}
d+k \\
i
\end{array}\right)}{2^{d+k}} .
$$

Equality is achieved if and only if $\mu$ is balanced about the origin. 
Remarks 3.8. 1. It can be shown that $\mu$ is balanced about the origin if and only if its radial projection $\breve{\mu}$ onto the unit sphere is symmetric about $\mathbf{0}$, i.e., invariant under reflection about $\mathbf{0}$. In dimension $d \leq 2$, this is rather trivial; for $d \geq 3$, we refer to Corollary 3.4 of [6].

2. The inequality (23) can also be derived by discrete methods, notably the UBT for $j$-facets (see Section 4), applied to successively larger random samples from the distribution $\mu$. This approach avoids the machinery of $h$-functions; it does not, however, lead to a characterization of the cases in which the upper bound is attained.

Upon further examination, more information can be squeezed from the proof of the CUBT. For instance, we say that a measure is a-balanced about an affine flat $\mathcal{A}, 0 \leq$ $a \leq \frac{1}{2}$, if every hyperplane containing $\mathcal{A}$ has mass at least $a$ on either side. Clearly, $\tilde{\mu}$ is $a$-balanced about $\tilde{\ell}$ iff $\mu$ is $a$-balanced about $\mathbf{0}$. Inductively, we see that the upper bound $h(y)=(d / 2) \min \{y, 1-y\}^{d-1}$ is attained for $y \leq a$ and $y \geq 1-a$. This gives, for example, lower bounds for $\tilde{s}_{k}(\mu, \mathbf{0})$ if the origin is a center point of $\mu$, i.e., if $\mu$ is $(1 /(d+1))$-balanced about $\mathbf{0}$.

\section{What about $h$-Vectors?}

You may wonder: What has all this got to do with the $h$-vectors of simplicial convex polytopes? Recall that these are defined as follows: if $\mathcal{P}$ is a $d$-dimensional simplicial polytope, and if $f_{k}=f_{k}(\mathcal{P})$ denotes the number of its $k$-dimensional faces, $-1 \leq k \leq$ $d-1$, then

$$
h_{j}=h_{j}(\mathcal{P}):=\sum_{k=0}^{j}(-1)^{j-k}\left(\begin{array}{l}
d-j \\
d-k
\end{array}\right) f_{k-1}(\mathcal{P}) .
$$

The sequence $\boldsymbol{h}:=\left(h_{0}, \ldots h_{d}\right)$ is called the $h$-vector of $\mathcal{P}$, and it proves to be a very convenient means of encoding the face numbers ${ }^{13} f_{k}$.

The reason why our $h$-functions can be thought of as continuous variants of $h$-vectors is the following connection between simplicial polytopes and $j$-facets of finite point sets. Let $S$ be a set of $n$ points in general position in $\mathbb{R}^{d}$. A $j$-facet of $S$, for integer $j$, is an oriented simplex $\sigma$ spanned by an ordered tuple of $d$ distinct points in $S$ such that exactly $j$ points of $S$ lie on the positive side of $\sigma$. Given a generic directed line $\ell$ (i.e., one which is disjoint from the convex hull of any $d-1$ points in $S$ ), we count the number of $j$-facets of $S$ that are entered by $\ell$; the resulting number is denoted by $h_{j}(S, \ell)$, and $\boldsymbol{h}=\left(h_{0}(S, \ell), \ldots, h_{n-d}(S, \ell)\right)$ is called the $h$-vector of $S$ and $\ell$.

Here is the promised connection, see [7]. It is based on a duality closely related to the Gale transform or LP-duality.

\section{Theorem 4.1.}

1. If $\boldsymbol{h}(\mathcal{P})$ is the h-vector of a simplicial d-polytope $\mathcal{P}$ on $f_{0}(\mathcal{P})=n$ vertices, then

13 These can be recovered from the $h_{j}$ 's since (24) just states that $\boldsymbol{h}=T \cdot \boldsymbol{f}$, where $\boldsymbol{f}=\left(f_{-1}, \ldots, f_{d-1}\right)$ and $T$ is the invertible matrix with entries $(-1)^{j-k}\left(\begin{array}{l}d-j \\ d-k\end{array}\right)$ (see [9]). 
there are a set $S$ of $n$ points in general position in $\mathbb{R}^{n-d}$ and a generic directed line $\ell \subset \mathbb{R}^{n-d}$ such that $\boldsymbol{h}(S, \ell)=\boldsymbol{h}(\mathcal{P})$.

2. Conversely, if $S$ is a set of $n \geq d$ points in general position in $\mathbb{R}^{d}$, and if $\ell \subseteq \mathbb{R}^{d}$ is a generic directed line which intersects $\operatorname{conv}(S)$, then there is some simplicial $(n-d)$-polytope $\mathcal{P}$ on at most $n$ vertices such that $\boldsymbol{h}(\mathcal{P})=\boldsymbol{h}(S, \ell)$.

Thus, we have a dictionary between "polytope-speak" and " $j$-facet-entered-by-a-linespeak." This guarantees that whatever results are known in one setting will be immediately applicable to the other. Examples are the Dehn-Sommerville relations

$$
h_{j}(S, \ell)=h_{n-d-j}(S, \ell)
$$

which just state that any directed line $\ell$ leaves as many $j$-facets as it enters, and the Upper Bound Theorem (UBT):

$$
h_{j}(S, \ell) \leq \min \left\{\left(\begin{array}{c}
j+d-1 \\
d-1
\end{array}\right),\left(\begin{array}{c}
n-j-1 \\
d-1
\end{array}\right)\right\} .
$$

Remark 4.2. At this point, the reader is perhaps curious to see a "discrete equivalent" of Corollary 3.7. For instance, one can show that given $n$ points in general position in $\mathbb{R}^{d}$, the number of $d$-simplices containing the origin in their interior is at most

$$
\left(\begin{array}{c}
\left\lfloor\frac{n+d}{2}\right\rfloor \\
d+1
\end{array}\right)+\left(\begin{array}{c}
\left\lceil\frac{n+d}{2}\right\rceil \\
d+1
\end{array}\right)
$$

Without bothering the reader with the details, we would also like to mention that via the duality indicated above, the probabilities $\tilde{s}_{k}$ can be thought of as continuous versions of $f$-vectors of simplicial polytopes (see [7]).

All in all, we see that $h$-functions are, in a sense, the natural extension of $h$-vectors to the continuous realm. Currently, we are investigating ${ }^{14}$ continuous analogues of the Generalized Lower Bound Theorem and the g-Theorem, see Section 8.6 of [9].

\section{Discussion}

We have analyzed the probability of the convex hull of $n$ random points (i.i.d. $\sim \mu$ ) to contain the origin. For that we borrowed the seminal concept of $h$-vectors from polytope theory, and introduced their continuous counterparts, $h$-functions. Note, however, that our proof of the upper bound on $h(y)$ is not merely a translation of McMullen's proof in the discrete setting [4]. Rather, when employed directly to the polytope set-up (via the duality discussed in [7]), it would also give a proof by induction of the UBT, but with the induction step going from $d+1 \rightarrow d$ and base case $d=n-1$.

\footnotetext{
${ }^{14}$ A note on how to derive the continuous Generalized Lower Bound Theorem from the discrete one can be found at www.inf.ethz.ch/ uli.
} 
Here is a challenging related open question. For $\mu$ on $\mathbb{R}^{2}$ given, consider

$$
a(\mu)=\operatorname{Prob}\left[P_{1} \in \operatorname{conv}\left\{P_{2}, P_{3}, P_{4}\right\}\right]
$$

for points $P_{1}, P_{2}, P_{3}, P_{4}$ i.i.d. $\sim \mu$. What is a tight upper bound for this probability over all (a.c.) probability distributions $\mu$ on $\mathbb{R}^{2}$ ? The probability $a(\mu)$ determines

$$
b(\mu)=\operatorname{Prob}\left[\operatorname{conv}\left\{P_{1}, P_{2}\right\} \cap \operatorname{conv}\left\{P_{3}, P_{4}\right\} \neq \emptyset\right]
$$

by the relation $4 a(\mu)+3 b(\mu)=1$. Hence, it is closely connected to the problem of determining a good bound for the crossing number of straight-line embeddings of complete graphs, see [5].

\section{Acknowledgment}

We are indebted to Rolf Schneider for many helpful remarks and in particular for bringing reference [6] to our attention.

\section{References}

1. Noga Alon and Joel Spencer. The Probabilistic Method. Wiley, New York, 1992.

2. Richard Durrett. Probability: Theory and Examples, 2nd edition. Wadsworth, Belmont, CA, 1996.

3. Gerald B. Folland. Real Analysis: Modern Techniques and Their Applications, 2nd edition. Wiley-Interscience, New York, 1999.

4. Peter McMullen. The maximum numbers of faces of a convex polytope. Mathematika (London), 17:179$184,1970$.

5. Edward R. Scheinerman and Herbert S. Wilf. The rectilinear crossing number of a complete graph and Sylvester's "Four Point Problem" of geometric probability. American Mathematical Monthly, 101(10):939943, 1994.

6. Rolf Schneider. Über eine Integralgleichung in der Theorie der konvexen Körper. Mathematische Nachrichten, 44:55-75, 1970.

7. Emo Welzl. Entering and leaving j-facets. Discrete \& Computational Geometry, 25:351-364, 2001.

8. James G. Wendel. A problem in geometric probability. Mathematics Scandinavia, 11:109-111, 1962.

9. Günter M. Ziegler. Lectures on Polytopes. Springer-Verlag, Berlin, 1994.

Received April 14, 2000, and in revised form October 6, 2000. Online publication June 20, 2001. 